\title{
Groundbreaking implant care and maintenance solutions
}

At EuroPerio9 in Amsterdam, Nobel

Biocare has announced a global exclusive partnership agreement with GalvoSurge Dental AG, a Swiss-based manufacturer of dental devices. The two companies intend to bring to market a new and innovative cleaning system for long-term implant maintenance on all major implant brands.

GalvoSurge Dental Implant Cleaning System provides a unique protocol for decontamination of dental implants by removing the bacterial biofilm attachment directly from the implant surface. Most other methods available today attempt to clean implants from the outside in, which might be challenging due to the macro and micro geometry of the implant. The system (which has been under development since 2012, and will be available in 2019) is based on an electrolytic process that activates the production of hydrogen, which lifts off the bacterial film. The groundbreaking process aims to be atraumatic and pain-free, only take 2-3 minutes per implant and maintain the implant surface integrity.

Hans Geiselhöringer, President, Nobel Biocare said: 'With the increasing number of patients receiving dental implant treatment, the importance of long-term maintenance is becoming more important. Following an intensive collaboration with leading clinicians who developed GalvoSurge, customers of all major implant brands will soon have additional means to provide lasting implant care for their patients. With this new partnership, we are again at the forefront of innovation in the dental industry.'

GalvoSurge inventors and internationally renowned clinicians and researchers commented:

Dr Markus Schlee explained: 'This innovative technology is unique, fascinating and one step ahead of other technologies and protocols.'

More information is available at nobelbiocare.com and galvosurge.com.

\section{Oral health - where do we go from here?}

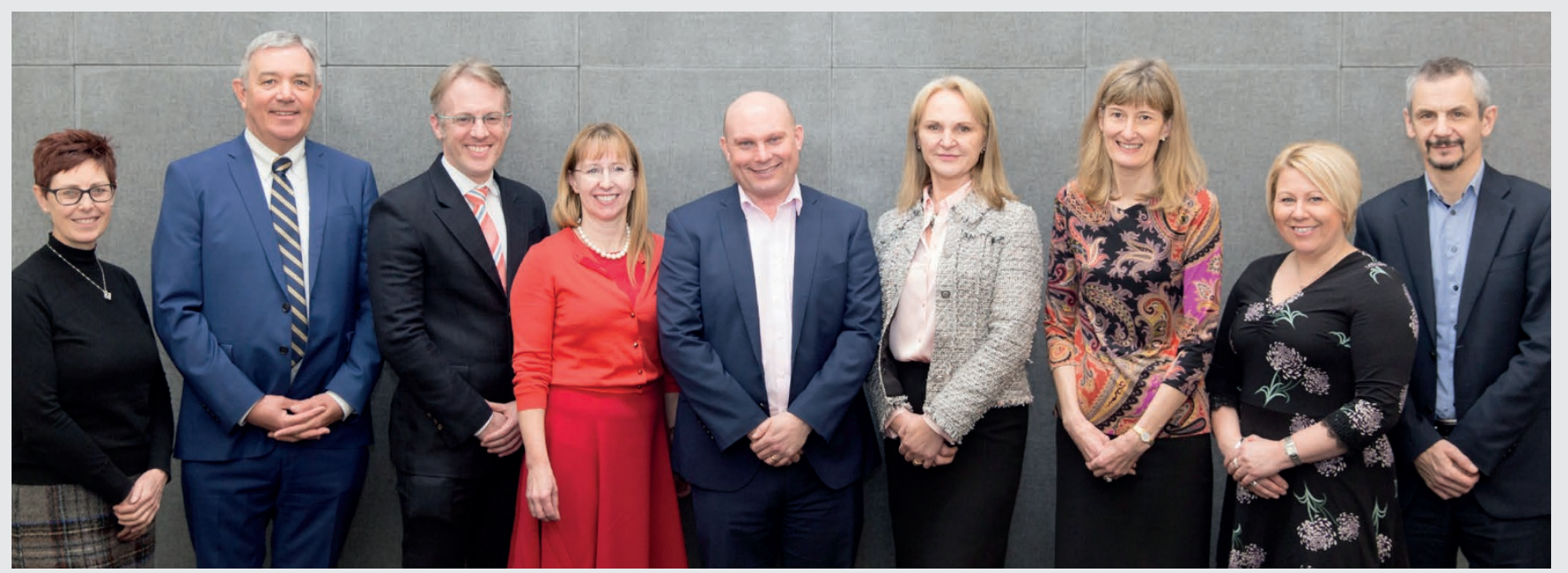

On 15 March, Johnson \& Johnson hosted The OH! Panel at the British Dental Association in London.

Chaired by Stephen Hancocks, it brought together eight key opinion leaders in dentistry: Ben Atkins; Julie Deverick; Penny Hodge; Tim Newton; Anthony Roberts; Julie Rosse; Nicola West; and Helen Whelton. Each was selected for their ability to provide unique insight into how the dental profession can be best supported going forwards, given the current oral health landscape.

A vision was agreed by The Panel as something all dental professionals should work by to help improve patients' oral health:

'The ultimate outcome is to improve oral health and therefore systemic health. The vision is that every dental healthcare professional, upon seeing a patient with gingivitis and/or periodontitis, can and will:

- Make a diagnosis(es) and communicate the relevance of the condition to the patient.

Explore risk factors and modify behaviour for successful outcomes

- Help every patient who receives the diagnosis(es) to be empowered to improve their oral health for life.'

Julie Deverick commented: 'I'm excited about the vision in the statement, because it's something we can all now bring to our societies and to our profession.'

Johnson \& Johnson have an ongoing commitment to the vision statement, and will work with that in mind, ensuring that all professional communications support the concept, to the benefit of the profession and patients.

The $\mathrm{OH}$ ! Panel was a natural extension of The $\mathrm{OH}$ ! Challenge, which was launched at the 2017 British Dental Conference and Exhibition. At the event, dental healthcare professionals (DHCPs) were invited to undertake a simple survey, created to test their knowledge in relation to key oral health topics. This provided valuable data for the creation of a communications programme to support DHCPs in keeping their knowledge current.

Please visit www.listerineprofessional. co.uk to see the results in more detail, to take the test and to access the supporting programme of evidence-based content. 